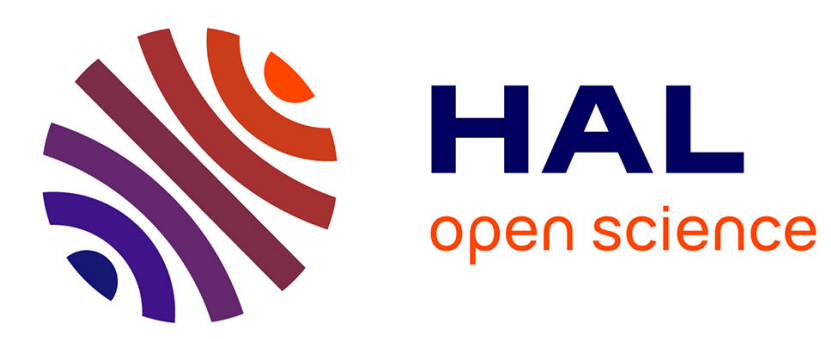

\title{
La viticulture en Gaule à l'âge du Fer
}

Michel Py, Ramon Buxó I Capdevila

\section{To cite this version:}

Michel Py, Ramon Buxó I Capdevila. La viticulture en Gaule à l'âge du Fer. Gallia - Archéologie de la France antique, 2001, La viticulture en Gaule, 58, pp.29-43. 10.3406/galia.2001.3172 . hal-01910098

\section{HAL Id: hal-01910098 \\ https://hal.science/hal-01910098}

Submitted on 20 Jan 2020

HAL is a multi-disciplinary open access archive for the deposit and dissemination of scientific research documents, whether they are published or not. The documents may come from teaching and research institutions in France or abroad, or from public or private research centers.
L'archive ouverte pluridisciplinaire HAL, est destinée au dépôt et à la diffusion de documents scientifiques de niveau recherche, publiés ou non, émanant des établissements d'enseignement et de recherche français ou étrangers, des laboratoires publics ou privés.

\section{(이)(\$)}

Distributed under a Creative Commons Attribution - NonCommercial - NoDerivatives| 4.0 


\title{
LA VITICULTURE EN GAULE À L'ÂGE DU FER
}

\author{
Michel Py et Ramon Buxó I CAPDEVILA
}

\begin{abstract}
Mots-clés. Archéobotanique, viticulture indigène, Marseille, Martigues, Lattes, outillage, dolium, amphores massaliotes.
Key-words. Archaeobotany, local viticulture, Marseille, Martigues, Lattes, implements, dolium, massaliote amphorae.

Résumé. Les rares sources littéraires disponibles n'apportent guère de témoignage d'une viticulture dans la Gaule du Nord, mais affirment en revanche son existence dans le sud.

Les sources archéologiques se placent dans le Midi surtout, notamment dans le territoire de Marseille, mais aussi en Provence et en Languedoc, avec la découverte de pépins de raisin, de pollens et de bois de vigne, de traces agraires, d'outils et de dolia. Sur le plan économique, Marseille produit du vin pour le vendre, s'opposant aux sites viticoles indigènes dont la production est très faible. Lattes représente un cas à part avec une viticulture extensive aux III-Ir s. avant notre ère.
\end{abstract}

\begin{abstract}
In the few available literary sources there is no evidence about the viticulture in Northern Gaul, but in the other hand we have accounts of its existence in the southern areas.

Archaeological sources are mainly situated in the South, around Marseille, but also in Provence and Languedoc, where thereare discoveries of pips, pollens, vine-shoots, land marks, implements and dolia. On the economical point of view, Marseille produces wine for exportation contrary to indigenous viticultural siteshaving a very low production. Lattes is a special case where we find an extensive vine cultivation during the $3^{\text {rd }}-1^{s t}$ centuries $B C$.
\end{abstract}

La question de la viticulture et de la production de vin en Gaule préromaine a été soulevée assez tôt mais rarement traitée de manière objective ${ }^{19}$. Du fait de la connotation éminemment symbolique de la plante et du produit, les archéologues et les historiens ont longtemps abordé le sujct sur lc plan culturel, se démarquant parfois

19. Voir principalement André, 1954 ; Dion, 1959 ; Benoit, 1962, 1965, p. 202-203. La prise en compte d'une viticulture gauloise préromaine a cependant longtemps reposé presque uniquement sur les mentions littéraires et mise loin derrière la consommation de vin grec, étrusque et italien. J. Jannoray, dans sa thèse, n'en parlait pas et a dû rajouter quelques mentions du bout des lèvres à la suite des remarques de son jury (Jannoray, 1955, p. 330, entre crochets) et B. Bouloumié écrivait encore en 1983 : « la vigne n'apparaît pas en Gaule - et encore s'agit-il de la seule Gaule méridionale - avant le ${ }^{\mathrm{er}} \mathrm{s}$. avant J.-C. " (Bouloumié, 1983a, p. 20). assez peu des auteurs de l'Antiquité classique pour qui vigne et vin, à l'instar d'olivier et huile, étaient signes de " civilisation » s'opposant à la bière et aux graisses barbares.

Pour autant, le dossier de la viticulture gauloise s'avère à l'examen assez varié, tant du point de vue des sources (littéraires, archéologiques, botaniques) que des lieux d'interrogation, selon que l'on se place aux environs de Marseille grecque, en Gaule méditerranéenne ou en Gaule continentale, ou bien selon les périodes envisagées, entre la Préhistoire et la colonisation romaine. Pour être complet, il faudra encore distinguer attestation de la vigne et viticulture, mais aussi production de raisin et vinification - en quelque sorte viticulture et viniculture.

On sait que la vigne est quasiment indigène sous forme sauvage (Vitis vinifera L. subsp. sylvestris) en 
Méditerranée occidentale ${ }^{20}$. On en retrouve sur le littoral des restes dès le Paléolithique (Nice, Terra Amata, cf. Boone, Renault-Miskovsky, 1976), puis dans plusieurs gisements mésolithiques et néolithiques ${ }^{21}$. Les attestations de pépins de raisin sauvage persistent, en France méditerranéenne, jusqu'à la fin de l'âge du Bronze (Marinval, 1997, p. 147). Plus à l'intérieur, la vigne sauvage est encore présente au début du deuxième âge du Fer, comme au Cluzel (Toulouse, Haute-Garonne) vers 400-350 avant notre ère (Marinval, 1988a). Envisager une longue tradition de cueillette du fruit de la lambrusque est donc possible, bien que l'extrême dispersion des restes laisse un doute sur la régularité de cette pratique.

Ce n'est en fait qu'à partir du milieu de l'âge du Fer, et plus précisément du $\mathrm{V}^{\mathrm{c}} \mathrm{s}$., que la multiplication des découvertes de pépins de raisin dans les gisements archéologiques a conduit à envisager une consommation régulière et une mise en culture.

Plusieurs questions restent cependant posées concernant l'origine de cette arboriculture. L'analyse de la morphologie des pépins et de leurs mensurations a fait apparaître certains caractères qui ont été jugés parfois intermédiaires entre la variété sauvage précédemment cueillie et la vigne cultivée (Vitis vinifera L. subsp. sativa) (Marinval, 1988a), telles qu'en témoignent pour l'Antiquité les études botaniques menées dans le monde classique (Renfrew, 1973) ; cette observation a suggéré l'hypothèse d'une amélioration locale de plantes indigènes ${ }^{22}$. Mais par ailleurs la chronologie des découvertes, et une tradition historique bien ancrée ont couramment porté les chercheurs à attribuer cette innovation aux influences coloniales méditerranéennes, phéniciennes dans le sud de l'Espagne, grecques ailleurs.

\section{LA TRADITION HISTORIQUE}

Les termes du débat imposent d'abord d'examiner les sources littéraires dans le cadre de la Gaule. De fait, le

20. C'est ce qu'indique notamment la palynologie : voir par exemple les analyses réalisées à Marsillargues, sur les bords de l'étang de Mauguio (Hérault), confirmant l'indigénat de Vitis vinifera subsp. sylvestris en languedoc (Planchais, 1982).

21. Par exemple à la Baume de l'Abeurador dans l'Hérault (Vaquer $e$ al., 1986), à Fontbrégoua dans le Var ou à Font-Juvénal dans l'Aude (Marinval, 1988a).

22. L'idée est déjà exprimée par Jullian, 1908, p. 270, qui attribuc la pratique aux Grecs. nombre de références concernant la viticulture indigène est considérablement plus faible que celui concernant la viticulture grecque et surtout la vente de vin par les Grecs et les Italiens aux populations locales. En vérité, on ne peut retenir sur ce point que deux allusions directes et une indirecte, l'une concernant le Midi, les autres le reste de la Gaule.

Pour le Midi, il s'agit du texte bien connu de Justin, résumant en une phrase les bienfaits de la colonisation phocéenne:

"Sous l'influence des Phocéens, les Gaulois adoucirent et quittèrent leur barbarie et apprirent à mener une vie plus douce, à cultiver la terre et à entourer les villes de remparts. Ils s'habituèrent aussi à vivre sous l'empire des lois plutôt que sous celui des armes, à tailler la vigne et à planter l'olivier, et le progrès des hommes et des choses fut si brillant qu'il semblait, non que la Grèce eût émigré en Gaule, mais que la Gaule eût passé dans la Grèce " (Justin, Abrégé des Philippiques, LXIII, 4, 1-2, trad. E. Chambry et L. Thély-Chambry).

Ce passage ne cite pas à proprement parler la production de vin, mais la taille de la vigne, et non la greffe, comme traduisent certains (par ex. Jannoray, 1955, p. 327, note 2) ce qui n'a pas empêché les historiens modernes d'extrapoler ${ }^{23}$. Le texte, bien que de date récente (II' $\mathbf{s}$. de notre ère), se réfère à des sources plus anciennes (Trogue-Pompée et au-delà) ; la mention de l'olivier, qui ne pousse en France qu'en contexte méditerranéen, limite la portée géographique de l'allusion au Midi.

Pour le Nord, on se référera à Diodore de Sicile, décrivant l'amour du vin des Gaulois au temps où les marchands romains envahissent la Gaule interne (fin II'début ${ }^{\mathrm{er}} \mathrm{s}$. avant notre ère) :

«L'excès du froid détruisant le caractère tempéré du climat, le pays ne produit ni vin ni huile. Ainsi ceux des Gaulois qui sont privés de ces produits préparent à partir de l'orge une boisson qu'on appelle zythos et usent également du jus provenant du lavage des cires d'abeille. Mais, aimant à la passion le vin, ils s'emplissent de celui qu'apportent les marchands sans le mélanger d'eau " ${ }^{24}$.

23. Ainsi par exemple Clavel-Lévêque, 1977, p. 170: "Le témoignage de Justin est formel : ce sont les Grecs qui ont appris aux barbares la taille, et sans doute avec elle l'ensemble des procédés si délicats de la culture et des méthodes de vinification ".

24. Diodore de Sicile, V, 26, 2-3 (trad. Lerat, 1977). Sur ce passage et le suivant, voir notamment les commentaires de Tchernia, 1986a, p. 88. 
Dans ce texte, où le climat rigoureux indique un cadre géographique continental, quatre choses concernent notre propos: premièrement, les Gaulois de cette zone ne produisent pas de vin ni d'huile (indirectement cependant, la formule " ceux des Gaulois qui en sont privés " suppose que d'autres Gaulois - et ce ne peut être que ceux du Midi - possédaient une viticulture) ; deuxièmement, comme boisson, ils produisent une bière d'orge (dénommée $\zeta \hat{v} \theta 0 \varsigma$ ) et de l'hydromel ; troisièmement, ils achètent leur vin à des marchands ; enfin, signe de barbarie, ils boivent ce vin pur et s'en enivrent.

Un texte d'Athénée, reprenant des indications de Poseidonios et décrivant donc une situation contemporaine (autour de 100 avant notre ère) ${ }^{25}$, confirme ces données et précise que le vin importé est réservé aux riches et qu'il provient d'Italie et de la chôra massaliète :

"Quant à la boisson, c'est, pour les riches, le vin qu'on fait venir d'Italie ou de la région marseillaise, d'ordinaire servi pur, parfois mêlé d'un peu d'eau ; pour les moins riches c'est la bière d'orge préparée parfois avec du miel, mais chez la plupart telle quelle: on l'appelle korma" (Athénée, Les Deipnosophistes, IV, 152, c-d, trad. Lerat, 1977).

Voici donc un tableau clair, opposant nettement le sud et le nord de la Gaule: d'un côté la "Gaule grecque " ${ }^{26}$, qui présente (mais depuis quand ?) tous les signes de l'hellénisation, parmi lesquels la culture de la vigne ; de l'autre la "Gaule barbare ", où l'on ne produit que de la bière ${ }^{27}$ et où l'on ne sait même pas boire de manière convenable (au sens "d'helléniquement correct ») le vin que l'on importe de Méditerranée.

Cette vision laisse bien entendu pleinement apparaître le «topos " de la supériorité des civilisations classiques et de leur rôle civilisateur, et l'on doit se demander quelle part de vérité se cache derrière ce discours ethno-

25. Poseidonios d'Apamée a séjourné en Gaule et notamment à Marseille à la charnière des $\mathrm{II}^{\mathrm{e}}$ et $\mathrm{I}^{\mathrm{er}} \mathrm{s}$. avant notre ère. Il a inspiré de nombreux auteurs postérieurs, parmi lesquels surtout Strabon (voir Lafranque, 1964, p. 79-85 ; Barruol, 1976, p. 14-15).

26. Selon la formule de Jacobsthal, Neuffer, 1933.

27. R. Dion (1959) avait déjà fait remarquer l'absence de toute référence à la viticulture dans les Commentaires de César, dont le théâtre d'opération ne descendit pas plus bas que les Cévennes; voir aussi Benoit, 1965, p. 203. centrique ${ }^{28}$. De fait les recherches archéologiques (au sens large du terme, incluant observations de fouille, études de mobiliers et analyses de restes) conduites depuis une cinquantaine d'années, si elles ont permis de renouveler quelque peu la documentation, ne sont pas venues contredire au fond ce que l'on avait déduit des textes: d'une part, le dossier de la viticulture en Gaule continentale reste à peu près vide ${ }^{29}$; d'autre part, il est de plus en plus certain qu'une viticulture s'est développée dans le Midi au cours de l'âge du Fer.

\section{PEU DE CHOSES EN GAULE INTERNE}

Hors du littoral méditerranéen et de la basse vallée du Rhône, on n'a en effet signalé aucune trace convaincante de mise en culture de la vigne avant la conquête romaine. Malgré l'explosion des recherches en milieu rural, favorisées par les fouilles préventives sur les grands tracés, peu de nouveautés sont apparues sur ce point. Citons quelques trouvailles de pépins de raisin apparemment cultivés signalés dans le domaine circum-méditerranéen, mais dans des contextes tardifs et particuliers : à Agen par exemple, il s'agit de trois fragments retrouvés dans le remplissage de trois amphores Dressel 1 immergées dans un puits, qui paraissent bien être venus avec les amphores ${ }^{30}$; à Feurs, de quelques pépins dans une tombe du Ir $\mathrm{s}$. Il ne semble pas encore y avoir là de quoi " ouvrir à nouveau le débat sur l'existence d'une viticulture gauloise " (Marinval, 1994, p. 50), sinon de manière " provocatrice ${ }^{31}$.

28. Bien analysé, en ce qui concerne la consommation de vin, par Dietler (1992), qui insiste sur le rôle social de l'alcool dans la société gauloise, et sur celui de la "fête " en tant qu'instrument politiquc, incomprise des gréco-latins et transcrite par eux comme une beuverie.

29. Constat présent dans Audouze, Buchenschut\%, 1989, p. 197 ; les auteurs écrivent : "De rares pépins de raisin attestent l'existence de la vigne sauvage, mais le vin reste un produit d'importation de très grand luxe ".

30. Le vin antique n'était pas soutiré et les amphores commercialisées contenaient fréquemment des restes de moût (pépins et fragments de rafle) : pour les amphores italiques, voir par exemple Tchernia, 1986a, p. 13 et note 4 ; pour les amphores de Marseille, Bertucchi, 1992, p. 189.

31. Selon le terme employé par Kaenel, 1985, p. 156, qui reconnaît d'ailleurs (p. 152) : "En Suisse occidentale, en Valais et sur les bords du Léman, aucune preuve archéologique ne peut être apportée en faveur de la culture de la vigne à la fin de La Tène déjà ". 
La documentation du Midi (Provence et Languedoc méditerranéen) est bien plus abondante, et de ce fait plus complexe, et méritera une attention particulière.

\section{LA VITICULTURE MASSALIÈTE}

S'il est en Gaule préromaine un producteur de vin reconnu, c'est bien Massalia ${ }^{32}$. Les Phocéens qui débarquent sur le Lacydon en 600 avant notre ère connaissent bien entendu de longue date la culture de la vigne et la vinification ${ }^{33}$. Mais le premier contingent, dont la composante aristocratique a été soulignée, venu s'installer dans le lointain Occident pour faire du commerce dans le cadre de l'emporia archaïque (Mele, 1986), ne s'adonne pas tout de suite à la culture de la vigne, du moins pour en faire commerce ${ }^{34}$. Les nouveaux arrivants achètent dans un premier temps le vin qu'ils boivent aux Étrusques de Vulci et de Caeré ${ }^{35}$, qui trafiquaient déjà sur les rivages gaulois depuis quelques années ${ }^{36}$. Ce n'est que plus tard, après la prise de Phocée (en 545) et la bataille d'Alalia (vers 540-535), qui provoquèrent sans doute l'arrivée de nouveaux groupes de colons (Gras, 1985, p. 403), et après quelques conquêtes territoriales aux dépens des indigènes locaux pour constituer une chôra suffisante, que les Massaliètes développent un vignoble non seulement pour leur propre consommation, mais également pour la vente à l'extérieur: en témoigne directement la création d'une amphore de

32. Un dossier assez complet concernant le vin marseillais de l'Antiquité au Moyen Âge a été rćuni par Bertucchi, 1992, p. 193-230.

33. (f. André, 1954, p. 363 sqq. Sur la viticulture et le vin en Orient et dans Ic monde grec archaique, of. Lutz, 1922; Dion, 1952 ; Milano, 1975 ; Rives, 1975 ; Marinval, 1997, p. 148-152.

34. Une production localc de consommation serait néanmoins attestée par l'abondance des pépins de raisin dès la première moitié du VI"s. dans les fouilles de la place Jules-Verne à Marseille (Marinval, 1997, p. 159). Sur ce point, voir supra, p. 24.

35. Voir les résultats des fouilles récentes dans les niveaux archaiques de Marscille, où dominent très largement les amphores étrusques (Gantès, 1992 ; Sourisseau, 1997).

36. Cf. Py, Py, 1974 et Py, 1985. Contra: Bats, 1998, mais avec des arguments bien peu convaincants. Si l'on suit la tradition de la fondation de Marseille, notamment le tcxtc d'Aristote (apud Athénée, XII, 576), le roi Nannos accueillant les Phocéens leur fait offrir par sa fillc " unc coupe de vin mêlée d'eau ", ce qui suppose que les indigènes connaissent le vin avant 600. Or l'archéologie démontre que dans cette région le vin en question ne peut être qu'ćtrusque. transport spécifique (l'amphore massaliète), qui connaît à partir du dernier quart du $\mathrm{VI}^{\mathrm{c}} \mathrm{s}$. une diffusion dans une vaste zone, et surtout (quantitativement) en Gaule du Sud (Py, 1978 ; Bats dir., 1990 ; Bertucchi, 1992).

Ce vignoble sera progressivement étendu à mesure du développement du territoire de Marseille (Bats, 1986a) ; au début du I ${ }^{\text {er }}$ s. de notre ère, Strabon peut écrire :

"Le pays est planté d'oliviers et couvert de vignes, mais il est très pauvre en blé à cause de son sol rocailleux ; aussi les Massaliètes ont-ils d'abord compté sur la mer plus que sur la terre, ct tiré parti, de préférence, des avantages naturels qui s'offrent à la navigation; mais plus tard, leur courage les rend assez forts pour ajouter à leurs possessions quelques-unes des terres qui les entourent " (Strabon, Géographie, IV, 1, 5, trad. Lasserre).

Dans cet extrait, Strabon rend compte du développement de l'agriculture marseillaise qu'il met clairement en liaison avec une extension de la chôra, mais il en souligne également les limites (des oliviers et surtout de la vigne ${ }^{37}$, mais peu de blé), ce qui permet probablement de repérer l'une des contreparties essentielles du commerce du vin marseillais: les céréales. Les traces agraires de tranchées de plantation et de provignage repérées dans la banlieue de Marseille au quartier de Saint-Jean-du-Désert, datables de la période hellénistique, sont venues récemment témoigner directement de ce vignoble (Boissinot, 1994 et infra, p. 55-58). La viticulture marseillaise est donc bien attestée, et la productivité peut en être évaluée par l'étude des conteneurs dans lesquels ce vin était distribué ${ }^{38}$.

Du point de vue technique, outre le provignage, on ne possède que des références assez disparates: vignes grimpantes, assez hautes pour y sculpter "une panthère ${ }^{39}$, qualité reconnue du vin marseillais : « un bon cru, épais et corsé " ${ }^{40}$, "le meilleur des Pyrénées aux Alpes ${ }^{41}$, avec plusieurs variétés: rouges plus

37. Cf. le sens renforcé que donne à katampelon le préfixe kata: Bats, 1988a, p. 31 ; Bertucchi, 1992, p. 199.

38. On pourra notamment se référer aux estimations du volume d'exportation vinaire de Marseille proposées pour le $\mathrm{IV}^{\mathrm{e}} \mathrm{s}$. avant notre ère à partir des épaves (Long, 1990) et aux chiffres de consommation d'une ville comme Lattes à la même époque (Py, 1999b).

39. Pline l'Ancien, Histoire naturelle, XIV, 9.

40. Athénée, Les Deipnosophistes, I, 27c.

41. Pline l'Ancien, Histoire naturelle, XIV, 68. 
ou moins forts (ibidem), blanc léger ${ }^{42}$, vin doux ${ }^{43}$. Insistons cependant sur le fait que toutes ces mentions concernent l'époque romaine ${ }^{44}$, et qu'il n'est pas évident qu'on puisse les transposer telles quelles à l'époque grecque $^{45}$.

Bien qu'assez documenté donc, le dossier de la viticulture marseillaise n'en laisse pas moins quelques zones d'ombre : on pense notamment à la gestion de la production et de la diffusion du vin par la cité, dont pourrait témoigner le système de marquage des amphores ; à la participation possible des colonies marseillaises à cette viticulture coloniale, en liaison avec la cadastration de leurs territoires ${ }^{46}$; ou bien encore au devenir de cette viticulture durant les années 125-50 avant notre ère, où Marseille ne fabrique apparemment plus d'amphores ${ }^{47}$...

\section{LA VITICULTURE INDIGÈNE EN GAULE MÉRIDIONALE À TRAVERS LES PÉPINS : ÉVIDENCES ET QUESTIONNEMENTS}

C'est à travers la présence répétée de pépins de raisin dans les niveaux archéologiques, et les caractères morphologiques de ces pépins (mensurations, rapport largeur/longueur, longueur du bec, rapport longucur du bec/longueur totale) renvoyant à Vitis vinifera, qu'a été valorisée ces dernières années la mise en place d'une viticulture indigène sur le littoral de la Gaule méditer-

42. Galien de Pergame, Thérapeutique à Glaucon, II, 372.

43. Martial, Épigrammes, XXIII, 42.

44. Sur la production marseillaise de vin à l'époque romaine, dont témoigne à la fois des séries spécifiques d'amphores et des inscriptions peintes sur celles-ci, voir Bertucchi, 1992, passim.

45. De même qu'il est illicite d'étendre à l'époque préromaine les indications que fournit Pline pour l'époque impériale, sur le vin doux des Voconces (Histoire naturelle, XIV, 83), le vin cuit des Helviens (XIV, 43) ou le vin poissé des Allobroges (XIV, 26-27), comme le fait Barruol, 1979 , p. 16.

46. Voir les études sur les cadastres d'Olbia (Benoit, 1985) et d'Agde (Benoit, 1978; Nickels, 1981 ; Clavel-Lévêque, 1982, 1989-1990). Concernant la viticulture agathoise préromaine, les données réunies par Garcia, 1995, p. 156 sont encore relativement faibles. L'absence de tout indice de production d'amphores agathoises limiterait d'ailleurs cette viticulture à une production de consommation.

47. Ce problème représente encore une énigme que ne résout vraisemblablement aucune des diverses propositions de Bats, 1986b, p. 406 . L'imitation par Marseille d'amphores Dressel 1, évoquée comme ranéenne (Buxó i Capdevila, 1996; Marinval, 1997, p. 144-145). Sans entrer dans l'analyse proprement carpologique du problème, traitée ailleurs ${ }^{48}$, il faut dire quelques mots de la signification archéologique de telles trouvailles, présentée souvent de manière imprécise par les études de botanique.

Les découvertes archéologiques de pépins peuvent avoir lieu dans différents contextes, mais l'immense majorité d'entre elles concerne des niveaux d'habitat, où ces restent se trouvent soit en position primaire (c'est le cas des sols, des sédimentations de sols, des fosses, des foyers, des entourages de foyers, voire parfois des fours), soit en position secondaire (par exemple dans les vidanges de foyers, les dépotoirs issus des nettoyages de sols ou de foyers, les comblements de silos de même origine, les remblais divers où se mêlent des rejets en provenance des maisons, etc.). Ces pépins sont le plus souvent conservés par carbonisation ${ }^{49}$. Dans de tels contextes, la carbonisation des pépins de raisin résulte du fonctionnement normal de l'habitat, et notamment des activités recourant à la combustion (cuisson, chauffage).

Un autre contexte de découverte est constitué par les niveaux d'incendie, plus complexes à analyser et qui réclament sur le terrain des stratégies d'échantillonnage spćcifiques. Ces niveaux sont en effet susceptibles de livrer, outre des documents assimilables au cas précédent (restes végétaux carbonisés lors de la vie du lieu, avant incendie), des concentrations particulières constituant des lieux clos: remplissage d'un récipient, tas de végétaux remisés dans une aire limitée, etc.

\footnotetext{
" seule alternative possible » n'est attestée pour l'instant que par le seul exemplaire du Marducl daté des dernières années du ${ }^{\mathrm{Ier}} \mathrm{s}$. avant notre ère (Py, Lebeaupin et al., 1986, p. 60, fig. 55, $\mathrm{n}^{\circ} 1$ ). Des imitations ponctuelles de Dressel 1B contemporaines sont par ailleurs attestées en Languedoc et dans la vallée du Rhône (Sabir et al., 1983; Laubenheimer et al., 1989); mais ces documents doivent se comprendre dans un contexte de romanisation, et non de production indigène. Sur le problème que pose l'usage de tonneaux par les Marseillais durant cette période de transition, que l'on pourrait fonder sur une allusion de César à des "cupae " remplies de poix et de branches de pin enflammées $(B . C ., \mathrm{Il}, 11,2)$, voir discussion dans Tchernia, 1997, p. 122.
}

48. Voir notamment Erroux, 1974, 1980a, 1987; Buxó i Capdevila, 1989, 1992, 1996 ; Marinval, 1988a, 1988b, 1988c, 1997 et la contribution de ce dernier auteur dans ce même volume.

49. Ils peuvent aussi parfois être conservés par minéralisation, ou, en milieu humide, à l'ćtat naturel. 
Tabl. III - Principales découvertes de pépins de raisin en Gaule méridionale (échantillons de plus de 100 graines de plantes cultivées).

\begin{tabular}{|c|c|c|c|c|c|c|c|}
\hline Site (Département) & Zone ou couche & Date (av. n. è.) & Type de contexte & Céréales & Légumineuses & Vigne & \% Vigne \\
\hline Mailhac (Aude) & F.22 & 75 & incendie, dolium & 468 & 28 & 1 & $0,2 \%$ \\
\hline \multirow{3}{*}{$\begin{array}{l}\text { Pierredon, } \\
\text { Éguilles } \\
\text { (Bouches-du-Rhône) }\end{array}$} & $80-\mathrm{C} 03$ & $200-150$ & vidange de foyer & 161 & 40 & 2 & $1,0 \%$ \\
\hline & 80-C03 & $225-175$ & contenu d'un dolium & 21 & 157 & 6 & $3,3 \%$ \\
\hline & $81-032$ & $225-175$ & contenu d'un dolium & 210 & 1235 & 6 & $0,4 \%$ \\
\hline \multirow{6}{*}{$\begin{array}{l}\text { Martigues } \\
\text { (Bouches-du-Rhône) }\end{array}$} & salle A1 & $400-375$ & niveau d'incendie & 430 & 32 & 13 & $2,7 \%$ \\
\hline & salle A1 & $400-375$ & contenu d'un vase en torchis & 146 & & 7 & $4,6 \%$ \\
\hline & salle A1 & $400-375$ & contenu d'une amphore & 631 & 2 & 1 & $0,2 \%$ \\
\hline & salle A2 & $400-375$ & niveau d'incendie & 1 & 5 & 730 & $99,2 \%$ \\
\hline & salle A2 & $400-375$ & contenu d'un vase en torchis & 81 & 301 & 8 & $2,1 \%$ \\
\hline & salle A2 & $400-375$ & contenu d'un vase en torchis & 48 & 75 & 1 & $0,8 \%$ \\
\hline $\begin{array}{l}\text { Coudounèu, } \\
\text { Lançon-Provence } \\
\text { (Bouches-du-Rhône) }\end{array}$ & ensemble & $400-375$ & $\begin{array}{l}\text { dolium II-3 } \\
\text { plus épandages }\end{array}$ & 4235 & 3891 & 955 & $10,5 \%$ \\
\hline \multirow{5}{*}{$\begin{array}{l}\text { Le Marduel, } \\
\text { Saint-Bonnet-du-Gard } \\
\text { (Gard) }\end{array}$} & $121-19$ & $250-200$ & remblai dans une ruelle & 71 & 31 & 2 & $1,9 \%$ \\
\hline & $122-26$ & $450-440$ & niveau d'incendie & 417 & 12 & 57 & $11,7 \%$ \\
\hline & $11-26$ & $450-440$ & niveau d'incendie & 431 & 7 & 30 & $6,4 \%$ \\
\hline & $11-24$ & $450-440$ & niveau d'incendie & 474 & 5 & 21 & $4,2 \%$ \\
\hline & $1011-23$ & $475-450$ & remblai de terrasse & 161 & & 17 & $9,6 \%$ \\
\hline \multirow[t]{4}{*}{ Gailhan (Gard) } & $\mathrm{S} 2 \mathrm{C} 4$ & $500-350$ & remblai & 10500 & 46 & 7 & $0,1 \%$ \\
\hline & $\mathrm{S} 3 \mathrm{C} 5$ & $500-475$ & remblai d'habitat & 110 & 1 & 3 & $2,6 \%$ \\
\hline & S3C6 & $500-475$ & remblai ou sédimentation & 252 & 3 & 25 & $8,9 \%$ \\
\hline & $\mathrm{S} 3 \mathrm{C} 7$ & $500-475$ & remblai ou sédimentation & 195 & 1 & 15 & $7,1 \%$ \\
\hline \multirow[t]{14}{*}{ Lattes (Hérault) } & 1393 & $325-300$ & remblai & 109 & 1 & 19 & $14,7 \%$ \\
\hline & 1459 & $325-300$ & contenu d'un four & 133 & 1 & 2 & $1,5 \%$ \\
\hline & 1515 & $350-325$ & remblai & 81 & 3 & 50 & $37,3 \%$ \\
\hline & 1770 & $375-350$ & niveau d'incendie & 280 & 14 & 157 & $34,8 \%$ \\
\hline & 3195 & $125-75$ & sol d'habitation & 6 & & 241 & $97,6 \%$ \\
\hline & 4310 & $25-1$ & comblement de fosse & 3 & & 533 & $99,4 \%$ \\
\hline & 4330 & $225-200$ & remblai dans une cour & 6 & & 396 & $98,5 \%$ \\
\hline & 4645 & $300-250$ & vidange de foyers & 42 & & 80 & $65,6 \%$ \\
\hline & 5153 & $100-50$ & remblai à base de dépotoir & 2 & & 177 & $98,9 \%$ \\
\hline & 7241 & $350-325$ & remblai dans une cour & 172 & 4 & 81 & $31,5 \%$ \\
\hline & 7317 & $375-350$ & remblai dans une cour & 240 & 6 & 57 & $18,8 \%$ \\
\hline & 9130 & $175-125$ & vidange de foyer & 2 & & 154 & $98,7 \%$ \\
\hline & 15006-15013 & 50 & remplissage de puits & 28 & & 57990 & $99,9 \%$ \\
\hline & 17067 & $225-200$ & fosse-foyer & 95 & 8 & 55 & $34,8 \%$ \\
\hline
\end{tabular}


L'examen du contexte des principales découvertes de pépins de raisin publiées ces dernières années ${ }^{50}$, tant en Provence (Pierredon, Martigues, Coudounèu) qu'en Languedoc (Mailhac, Le Marduel, Gailhan, Lattes) révèle des situations relativement diverses (tabl. III).

Hormis le site de Lattes, sur lequel nous reviendrons en détail ci-après, la quasi-totalité des prélèvements fait apparaître une présence très minoritaire du raisin par rapport aux céréales et/ou aux légumineuses; les proportions dépassant rarement $10 \%$ du total des graines d'espèces cultivées, les deux tiers des cas étant compris entre 0,1 et $3,3 \%$. Cette observation est valable dans tous les types de contexte, qu'il s'agisse de sols ou de remblais d'habitat, de remplissages de vases ou de niveaux d'incendie.

Si la multiplication de telles attestations témoigne bien de l'existence d'une culture de la vigne dans la zone considérée (principalement pour l'heure le triangle basrhodanien) à partir des environs de 500 avant notre ère ${ }^{51}$, la faiblesse des proportions, et l'association assez systématique des pépins à des grains de céréales ont conduit à envisager plusieurs utilisations possibles du raisin cultivé : en tant que fruit frais ou fruit sec, ces derniers éventuellement mélangés aux grains pour des raisons diététiques, ou entrant dans diverses préparations (pain au raisin notamment); mais également transformation des pépins eux-mêmes en produits dérivés, tels que farine ou huile. Tous ces usages sont théoriquement possibles - car attestés par les sources et l'ethnographie (Maurizzio, 1932 ; André, 1981) -, mais peu d'entre eux bénéficient de confirmations archéologiques ${ }^{52}$.

Seuls, dans le groupe des prélèvements non lattois, deux échantillons s'individualisent; il s'agit dans les deux cas de restes brûlés lors d'un incendie. Le premier exemple provient de la maison A2 du premier village de l'île-de-Martigues, détruite lors de l'incendie qui a ravagé

50. Cet examen a été limité aux échantillons de plus de 100 graines permettant une approche statistique fiable. Dans cette approche, il convient d'éliminer des comptages les graines de mauvaises herbes, dont la signification est tout autre.

51. Et non pas " dès la fin du VII ${ }^{e}$ s. avant J.C.C. " comme l'écrit Marinval, 1997, p. 159, en nous attribuant fallacieusement cette opinion ( $c f . i b i d$., notes 198 et 199)!

52. On peut seulement rappeler les galettes dont la surface est parsemée de pépins de raisin (mais aussi de figues) découvertes dans un puits de Cavaillon, et datées du début $\mathrm{du}^{\mathrm{Ir}} \mathrm{s}$. de notre ère (cf. Dumoulin, 1965, p. 77-78). l'agglomération vers le milieu du V $\mathrm{V}^{\mathrm{e}} \mathrm{s}$. La composition de l'échantillon est relativement pure (Chausserie-Laprée, 1990 , p. 65), puisqu'à côté d'un grain d'orge et de 5 légumineuses, on a décompté 730 pépins de raisin qu'accompagnaient également 66 fragments de pédoncules de Vitis. Cette homogénéité rend probable l'hypothèse d'un déversement sur le sol de l'habitation d'un des nombreux conteneurs brisés lors de la destruction. Le second cas, provenant de la pièce II-3 de la ferme indigène de Coudounèu, confirme cette observation : ici, un grand nombre de pépins de raisin (à l'exclusion de tout autre type de graine) a été brûlé lors d'un incendie au début du $\mathrm{IV}^{\mathrm{e}} \mathrm{s}$. au fond d'un dolium (échantillon 31-1050) (Marinval, 1996-1997). La présence de pédoncules et de fragments de rafle témoigne de l'existence non seulement de fruits mais également de grappes, ce qui a fait penser à une réserve de raisins $\operatorname{secs}^{53}$.

Une autre hypothèse a été développée à partir des découvertes de Lattes. Dès l'origine des recherches menées sur le site, on a remarqué la fréquence des restes de vigne cultivée. Les sondages du Groupe archéologique Painlevé (GAP) ont révélé en effet dès 1964 de très nombreux pépins de raisin dans la couche $8-9 \mathrm{du}$ sondage GAP-3, datée du début du $\mathrm{V}^{\mathrm{e}} \mathrm{s}$. avant notre ère ; l'analyse qui en a été faite ne laissc aucun doutc sur l'appartenance à Vitis vinifera, et reconnaît deux variétés différentes (Erroux, 1974). L'année suivante, un " cône de pépins de raisins " attribué au $\mathrm{II}^{\mathrm{e}} \mathrm{s}$. avant notre ère est signalé dans le sondage GAP-4 ${ }^{\text {bis }}$ (Richard, 1973, p. 92). D'autres découvertes interviendront encore: dans le sondage GAP-16 en 1971, un canal comblé aux III ${ }^{\mathrm{C}} \mathrm{II}^{\mathrm{e}} \mathrm{s}$. livre une multitude de pépins et des restes de sarments (Py, 1988, p. 82) ; en 1984, dans le secteur 21 du sondage GAP-26, le puits 84-1 comblé au $\mathrm{II}^{\mathrm{e}} \mathrm{s}$. avant notre ère contient des amas de pépins carbonisés (Py, 1988, p. 105).

Ces premières indications ont été largement confirmées par les fouilles programmées entamées sur le site à partir de $1983^{54}$, qui ont permis de développer des prélèvements systématiques de macrorestes (Buxó

53. Sur la conservation des raisins secs en grappe (d'après le Traité d'agriculture de Palladius), cf. Blanc, Nercessian, 1992, p. 191.

54. Sur ces fouilles en général, voir une première synthèse dans $P y$, Garcia, 1993, et les comptes rendus réguliers publiés dans la série Lattara ( ${ }^{\circ *} 1-1988$ à 14-2001). 
i Capdevila, 1989). Or, parmi les échantillons prélevés, qui couvrent actuellement cinq siècles de la vie du site (de la fin du $V^{e} s$. avant notre ère au $I^{\text {er }} s$. de notre ère), un grand nombre livrent des pépins de raisin ${ }^{55}$. Parmi les cas statistiquement significatifs (contenant plus de 100 graines d'espèces cultivées) (tabl. III), le seul qui recèle une très faible proportion de vigne correspond au remplissage d'un four à pain ; les autres échantillons mixtes (céréales plus raisin) livrent entre 14 et $65 \%$ de pépins, tandis que six concentrations ne se composent presque que de pépins ( 97 à $99 \%$ des graines). Remarquable est l'amas retrouvé dans un puits du milieu du $\mathrm{I}^{\mathrm{er}} \mathrm{s}$. avant notre ère dans l'îlot 15 de la ville (PT615, Us 15006, 15011 et 15013), qui ne livre pas moins de 57990 pépins et une quarantaine de fruits entiers.

Cette abondance et les conditions de découverte, si elles confirment ici nettement la pratique de la viticulture, ne sont guère compatibles avec les interprétations précédemment retenues. En effet, la carbonisation d'un tel nombre de pépins, la plupart du temps en milieu domestique et sans qu'intervienne d'incendie ${ }^{56}$, ne semble pas pouvoir s'expliquer par la simple consommation du fruit. Si des pratiques de grillage et de torréfaction peuvent pour partie rendre compte de la présence régulière de céréales autour des foyers et dans leurs vidanges, le même raisonnement ne saurait en effet s'appliquer à la vigne. Seule dans ce cas, pensons-nous, l'utilisation courante de résidus de marc séché pour allumer ou entretenir le feu peut expliquer la fréquence des restes de pépins carbonisés (mais aussi de rafle), et l'existence de grands amas du type de celui du puits PT615. Or qui dit marc dit pressage, jus, et bien entendu vin.

C'est donc vers la prise en compte non seulement d'une viticulture mais également d'une viniculture indigène précoce et continue durant l'âge du Fer que nous conduit l'analyse des données carpologiques de Lattes.

55. Parmi les 124 échantillons étudiés dans Buxó i Capdevila, 1992, pas moins de 90 (soit $72 \%$ ) contiennent de la vigne.

56. Plusieurs niveaux d'incendies ponctuels, ayant affecté des maisons de l'habitat protohistorique de Lattes, ont été cependant retrouvés dans les fouilles récentes, notamment pour le IV ${ }^{\mathrm{c}} \mathrm{s}$. (Roux et al., 1996, p. 360 ; Buxó i Capdevila et al., 1996, p. 388-395; Lebeaupin, 1999, p. 139 sqq), ct les sćdiments ont aussi livré des restes de vigne, mais dans les mêmes proportions que les autres niveaux contemporains. Ces documents sont actuellement à l'étude.

\section{AUTRES INDICES POSSIBLES DE LA VITICULTURE INDIGENNE À L'ÂGE DU FER}

Trouve-t-on, dans les données sur l'âge du Fer méridional, d'autres arguments susceptibles de renforcer l'idée de l'existence et du développement d'une viticulture indigène avant l'époque romaine? Les documents utilisables sont à la vérité plus divers que nombreux, et de valeur inégale.

Parmi les restes végétaux, les pollens peuvent être mis à contribution, bien qu'il soit difficile de distinguer parmi eux Vitis vinifera de Vitis vinifera sylvestris. Dans la région de Lattes, les diagrammes polliniques réalisés aux environs de Palavas (Planchais et al., 1977; Planchais, 1987) et de Marsillargues (Planchais, Duzer, 1978 ; Planchais, 1982) ne traduisent qu'une faible augmentation de la présence de vigne dans la plaine littorale à l'âge du Fer et à l'époque romaine. Par contre, certains carottages effectués dans les environs immédiats de la ville de Lattara, notamment dans le secteur d'Embouchac, montrent une très forte augmentation des populations de Vitis et de Cerealia au Subatlantique, dans des proportions qui confirment nettement le développement de ces cultures au cours de la Protohistoire (Puertas, 1998, p. 35-42).

Les bois de vigne carbonisés ou gorgés d'eau (racines, ceps et sarments) apportent une indication supplémentaire. Des ceps de belle allure ont été recueillis dans le deuxième village de l'île-de-Martigues (II' ${ }^{\mathrm{s}}$ s. : Blanc, Nercessian, 1992, p. 42, fig. 38) et dans un puits d'Espeyran (Barruol, Py, 1978, p. 80-81 : époque augustéenne). Les charbons de bois recueillis dans l'habitat de Lattes confirment la présence de la vigne (Chabal, 1997a, p. 124) et montrent une présence particulièrement marquée dans les foyers domestiques du $\mathrm{IV}^{\mathrm{e}}$ et du $\mathrm{I}^{\mathrm{cr}} \mathbf{s}$. avant notre ère (Ambert, Chabal, 1992, p. 22, fig. 7). Enfin, un sondage dans les niveaux inondés du port de Lattes a fourni plusieurs fragments de ceps et de sarments conservés par l'eau dans un horizon du $\mathrm{I}^{\mathrm{er}} \mathrm{s}$. avant notre ère (Us 37015, cf. Chabal, 1997b, fig. 6).

Les traces agraires interprétables comme des plantations de vignes étaient jusqu'à présent situées principalement dans des environnements coloniaux. Nous avons rappelé ci-dessus celles de Marseille (Saint-Jean-duDésert) ; on peut mettre en parallèle, dans un contexte de romanisation, les petites parcelles de vigne suburbaines découvertes récemment à Nîmes et datables de 
la fin du II ${ }^{\mathrm{e}}$ et du début du I ${ }^{\mathrm{er}}$ s. avant notre ère, qui sont peut-être liées aux premières installations de colons italiens dans la cité (Monteil, 1999a ; Monteil et al., 1999).

Bien différents, et d'une autre ampleur, sont les vastes champs de vigne révélés en 1999 par une fouille d'urgence aux portes de Lattes, dans le quartier de PortAriane, à moins de $500 \mathrm{~m}$ des murs de la ville protohistorique. Ici, des réseaux de plantation des III $^{\mathrm{e}} \mathrm{I}^{\mathrm{Ir}} \mathrm{s}$. avant notre ère se succèdent et s'entrecroisent sur plusieurs milliers de mètres carrés, témoignant pour la première fois d'une véritable viticulture extensive d'âge préromain ${ }^{57}$.

Les outils que l'on peut raisonnablement relier à cette culture ne sont guère spécifiques : des pioches en fer ${ }^{58}$ sans doute pour le creusement des tranchées et des trous de plantation, des houes à lame rectangulaire ${ }^{59}$, à extrémité appointée ${ }^{60}$ ou à deux dents ${ }^{61}$ pour le déchaus-

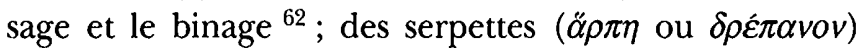
munies sur le dos d'un ergot ${ }^{63}$ ou d'une courte lame faisant hachette (falx vineatica) ${ }^{64}$, pour tailler la vigne, couper les rejetons et les chicots de bois mort, dont la forme est bien attestée par l'iconographie romaine du Midi, mais se rencontre déjà à l'âge du Fer; peut-être encore des serpettes de petite taille à lame recourbée

57. Fouille d'urgence menée durant l'été 1999 sous la direction d'Isabelle Daveau. Cf.Jung, 2000, notamment p. 367, fig. 10.

58 . Le $\mathrm{V}^{\mathrm{c}} \mathrm{s}$. avant notre ère connaît en Languedoc oriental des pics à douille en fer et emmanchement coudé (Tendille, 1982, fig. 5, $\mathrm{n}^{\circ \times} 32-34 ;$ Py, 1990b, doc. 87, $\left.\mathrm{n}^{\mathrm{os}} 1-2\right)$, dont la date d'apparition coincide avec celle de la vigne cultivée. On en connaît aussi une variante à soie (Py, 1993, p. 219, fig. 41, en bas à gauche). Plus tard, au deuxième âge du Fer, sont attestés des pics à trou d'emmanchement de forme plus "moderne " (Py, 1979, p. 87, n 21 et p. 89, nos 4-10; Py, Garcia, 1993, p. 54 , fig. $35, \mathrm{n}^{\text {os }} 6-7$ ).

59. Voir des exemplaires provençaux et languedociens, datables pour la plupart des phases récentes de l'âge du Fer, dans Py, 1979, p. 89, $\mathrm{n}^{\circ} 13$; Tendille, 1982, fig. $5, \mathrm{n}^{\text {os }} 5-36$; Py, 1990b, doc. 87 , $\mathrm{n}^{\text {os }} 3$ et 5 ; Boissinot, 1990 , p. $97, n^{\circ} 47$.

60. Py, 1979, p. 89, $\mathrm{n}^{\circ} 15$; Boissinot, 1990, p. $97, \mathrm{n}^{\circ} 45$.

61. Py, 1979, p. 89, nº 14 ; Boissinot, 1990, p. 97, nº 46.

62. Sur l'usage de la houe dans l'agriculture traditionnelle, $c f$. Benoit, 1947, p. 23-29 ; Leroi-Gourhan, 1973, p. 120-124.

63. Py, 1979, p. 87, ${ }^{\circ} 13$; Tendille, 1982, fig. $6, \mathrm{n}^{\circ} 40$.

64. Py, 1979, p. $87, \mathrm{n}^{\text {os }} 9,14,18$; Tendille, 1982 , fig. 6 , $\mathrm{n}^{\text {os }} 39$ et 41 ; en général : Benoit, 1947 , p. $62-63$. Une minuscule serpette à hachette dorsale en argent a été recueillie à La Lagaste (Rancoule, 1980, p. 100, pl. $5, n^{\circ} 4$ et p. 119 , fig. $\left.57, n^{\circ} 4\right)$ : il s'agit vraisemblablement d'un objet votif. (falcula) pour vendanger ${ }^{65}$. Cependant, il est douteux que ces instruments aient été à cette époque strictement réservés au travail de la vigne, et il serait imprudent de déduire de leur répartition ${ }^{66}$ une carte de la viticulture.

La fabrication du vin n'a pas non plus laissé en Gaule $\mathrm{du}$ Sud d'installations aussi claires que celles que l'on a signalées récemment dans le sud-est de l'Espagne pour l'époque ibérique ancienne ${ }^{67}$ : pas de fouloirs, de cuves ${ }^{68}$ ni autre aménagement fixe que l'on puisse clairement mettre en relation avec cette activité ${ }^{69}$. Les pressoirs en pierre des $\mathrm{IV}^{\mathrm{c}}-\mathrm{I}^{\mathrm{er}}$ s. signalés en Provence (Brun, 1984, 1986, 1987 ; Brun et al., 1998) et en Languedoc (Garcia, 1992a), par leur morphologie, se rapportent avec évidence pour la plupart $^{70}$ à la production d'huile

65. Par exemple Py, 1979, p. 87, $\mathrm{n}^{\mathrm{os}}$ 3, 4, 5, 8 et 16 ; Py, Garcia, 1993, p. 54 , fig. $35, \mathrm{n}^{\circ} 4$.

66. P. Marinval, 1994, p. 50 cite la thèse de Coutel, 1992, que nous n'avons pas pu consulter, et qui s'appuierait sur la typologie des serpettes retrouvées en Europe pour soutenir l'existence d'une vaste expansion d'une viticulture " artisanale " (?) avant la conquête romaine. Mais l'on sait par ailleurs l'ambiguité de cet outil multi-usages durant la Protohistoire où, en l'absence de faucilles, il servit très probablement à moissonner les céréales à la gerbe ( $c f$. Py, 1990b, p. 425-428).

67. Des recherches récentes ont confirmé pour l'est de l'Espagne la présence de structures associées à la vinification dès le $\mathrm{VI}^{\mathrm{c}} \mathrm{s}$. avant notre ère à l'Alt de Benimaquia (Dènia) (Gómez Bellard et al., 1993a, 1993b ; Gómez Bellard, Guérin, 1994). Selon les fouilleurs, la capacité de production de ces structures dépasse largement les besoins d'une consommation locale, et fait donc penser à une culture spéculative, dont le produit aurait été diffusé dans des amphores fabriquées sur place et imitant des prototypes phéniciens.

68. Rappelons cependant la découverte à Lattes, dans un puits d'époque augustéenne, d'une sorte de cuve de bois cerclée de forme cylindrique (qui ne présente pas la courbure d'un tonneau), et qui peut correspondre aux "conteneurs de bois cerclés " que Pline l'Ancien (H. N., XIV, 132) signale dans les Alpes (Py, 1988, p. 101, fig. 21 et p. 102, fig. 22). Il n'est pas inintéressant de relever que ce puits a livré toute une panoplie de témoins en relation possible avec le vin : carporestes de vigne, cuve de bois, dulium (servant de margelle) et amphores...

69. La situation du sud de la Gaule n'est pas en ce domaine unique : voir par exemple les remarques de C. Vandermersch (1994, p. 34-36) sur l'indigence des traces de fabrication du vin en Italie du Sud et en Sicile pour les IV $\mathrm{V}^{\mathrm{c}}-\mathrm{III}^{\mathrm{c}} \mathrm{s}$., régions et époque où une viticulture intensive est attestée par les textes et les fabrications d'amphores; l'auteur oppose cette indigence à la richesse des informations fournies par les fouilles de la mer Noire.

70. J.-P. Brun a émis un doute quant à l'utilisation oléicole des maies polygonales d'Agde, d'Ensérune et de Magalas présentées par Garcia (1992a), qu'il rapproche des tables de presse viticoles des stèles des Hermocopides (Brun, 1993a ; Garcia, 1995, p. 156). 
d'olive, dont le développement à l'âge du Fer, parallèle à celui de la viticulture, a certainement été en partie sousestimé $^{71}$.

Par contre, il ne fait guère de doute que le pithos ou dolium, grosse jarre dont la fonction principale fut de stocker le grain ${ }^{72}$, a effectivement pu servir de cuve de fermentation et de stockage en vrac pour le vin. Outre une tradition littéraire bien documentée dans le monde classique $^{73}$, plusieurs indices archéologiques - en attendant d'éventuelles analyses tracéologiques - en portent témoignage, notamment des timbres et des inscriptions.

Ce type de vaisseau, apparemment ignoré dans le monde celtique continental jusqu'à la conquête, mais aussi en Gaule méridionale avant le milieu du $\mathrm{VI}^{\mathrm{e}} \mathrm{s}$., a probablement été emprunté par les indigènes aux Phocéens de Marseille, qui en possèdent dès le début du $\mathrm{VI}^{\mathrm{e}} \mathrm{s}^{74}$. Bien que de nombreuses découvertes de dolia contenant du grain indiquent pour utilisation principale la conservation des céréales, on ne peut s'empêcher de remarquer que le premier développement de l'usage de cet ustensile sur les sites littoraux, qui date des environs de 500, est contemporain de l'apparition des pépins de raisin cultivés.

Les timbres doliaires et les inscriptions gravées sur des dolia $\mathrm{des}_{\mathrm{III}} \mathrm{e}^{\mathrm{e}} \mathrm{II}^{\mathrm{c}} \mathrm{s}$. avant notre ère retrouvés en Languedoc.

71. Cf. L.cveau et al., 1991, notamment p. 87 et 91 . Signalons la découverte récente à Lattes d'un pressoir intact des environs de 400 avant notre ère, en position de fonctionnement dans une cour domestique de l'îlot 27 (Lebeaupin, 1999, p. 134, fig. 5). Dans un niveau contemporain du même îlot a été retrouvé un vase spécialisé dans la conservation et le service de l'huilc (grand lécythe globulaire en pâte claire massalic̀tc) qui renforce l'idée d'une consommation locale d'huile d'olive (Py, 1999 a, p. 175) : cet indice peut laisser penser que la répulsion des Celtes pour cette denrée dont parle Poseidonios (apud Athénée, 151) ne concernait pas tous les Gaulois.

72. Sur les dolia méridionaux, voir Tardicu, 1976; Py, 1990b, p. 406-407 et 428 ; Garcia, 1987 et 1992a.

73. La liaison intellectuclle entre pithos et vin est telle que Strabon parle, pour désigner les foudres ou les tomneaux, de " pithoi en bois" (Geographie, V, 1, 8; V, 1, 12). Pour la conscrvation du vin dans les dolia, voir par excmple l'expression "vinum bebere e dolio" de Pline l'Ancien, H. N., XIV, 89.

74. (j. Carcia, 1997, p. 90-91. Cette originc phocéenne du modèle, qu'il serait intéressant de valider par une étude typologique (mais il faudrait pour cela que les fouilleurs de Marseille commencent un jour à publier leurs données), n'est pas forcément exclusive, comme le montre la présence d'un fragment de pithos attique dans un niveau du $\mathrm{IV}^{\mathrm{e}} \mathrm{s}$. de Lattes (Py, 1996a, p. 97, fig. 20, $\mathrm{n}^{\circ} 20$ ). occidental et en Roussillon (Ensérune, Montlaurès, Pech-Maho, Ruscino) ont été depuis longtemps évoqués dans le sens d'une utilisation vinicole des jarres qui les portent. À côté de nombreux timbres représentant des grappes ${ }^{75}$, on relèvera un timbre plus original d'Ensérune figurant un personnage tenant une grappe et surmontant l'inscription ibérique «botiror » (Jannoray, 1955, p. 263 et pl. 14, n 1). Le même site a donné une inscription semblable gravée sur le col d'un dolium, que J. Jannoray n'a pas manqué de rapprocher du cas précédent. Un autre timbre doliaire d'Ensérune, comprenant deux petites grappes de raisin, comporte à droite l'inscription en relief "boture " (Tardieu, 1976, p. 39). Enfin un graffite ibérique sur dolium provenant de Pech-Maho, qui se lit «burur», peut être également cité. Ces vocables évoquent incontestablement une boisson, que la connexion avec les grappes invite à identifier avec le vin (Jannoray, 1955, p. 437). L'association des grappes de raisin et des épis de céréale sur certains timbres pourrait indiquer pour sa part que les dolia avaient, en Gaule du Sud comme ailleurs en Méditerranée, pour fonctions principales de conserver soit du grain, soit du vin (ibid., p. 262).

Une autre inscription tracée à cru et deux fois répétée sur un dolium de Saint-Blaise, dont la chronologie est discutée, est en lettres grecques et se lit MFPEIIKOD. Ce terme a été rapproché du grec $\mu v \rho \varepsilon \tau \sigma \iota k o ́ \varsigma$ qui signifie " (vin) parfumé » et mis en relation avec le stockage de vin dans le récipient ${ }^{76}$.

\section{ESSAI D'INTERPRÉTATION ÉCONOMIQUE ET SOCIALE DE L'ACTIVITÉ VITICOLE}

Une fois inventoriés les divers indices relatifs à la viticulture préromaine, il reste à s'interroger sur la place qu'occupait cette activité dans l'économie. Malgré l'indi-

75. Cf. Héléna, 1937, p. 302-303 et fig. 188-189; Jannoray, 1955, pl. 71, $n^{\text {os }} 4$-7. Les « chapiteaux ioniques " présents sur plusicurs de ces timbres pourraient évoquer des vrilles de vigne.

76. L.e fragment de dolium provient d'un nivcau hellénistique d'une maison proche de la porte méridionale du rempart, datation retenue par Arcelin, 1990, p. 203. H. Rolland (1951, p. 145-147) et G. Bertucchi (1992, p. 195-196) penchent pour une datation archaïque en sc fondant sur la forme des lettres. Notons que lc digamma, employé ici pour transcrire le $\mathrm{U}$, est encore utilisé au IV $\mathrm{V}^{\mathrm{e}} \mathrm{s}$. parmi les estampilles des amphores de Marseille (Bertucchi, 1992, p. 156). 
gence relative de nos informations actuelles (mais les données se multiplient rapidement), il paraît évident que cette importance peut être considérée comme différente selon les lieux.

Au cours de l'âge du Fer, le cas de Marseille s'individualise nettement par rapport aux autres zones par le fait que la production vinicole y apparaît comme une activité visant plus au commerce qu'à la consommation. Quel que soit le nombre considérable d'amphores micacées qu'ait livré ou que recèle le sol de la cité, la quantité de celles susceptibles d'être trouvées à l'extérieur est bien plus grande encore ${ }^{77}$. Tout laisse penser que la viticulture était pour Massalia, et plus spécialement pour sa classe dirigeante ${ }^{78}$, une source de revenus considérables ${ }^{79}$, probablement la source principale ${ }^{80}$.

Aucune donnée, ni textuelle ni archéologique, n'illustre avant l'époque romaine un tel processus hors de Marseille: nulle agglomération indigène de Gaule méridionale ne semble, dans l'état actuel des données, avoir fabriqué d'amphores qui auraient permis une commercialisation de sa production viticole. Le seul cas signalé d'imitations locales d'amphores massaliètes, qui est celui du Pègue ${ }^{81}$, se situe précisément sur un site qui n'a jamais livré de traces de culture de la vigne ${ }^{82}$.

77. On peut remarquer à ce propos que le niveau de consommation marseillais traduit par les proportions d'amphores dans les mobiliers archéologiques, si tant est qu'on puisse l'estimer à partir des chiffres de Gantès (1992), ne dépasse guère celui d'Olbia et paraît inférieur à celui de Lattes (Py, 1992, p. 327, fig. 11).

78. Sur la liaison entre l'accès aux décisions politiques et l'accès aux ressources du territoire dans la constitution marseillaise, $c$. Bats, 1986a, p. 24.

79. Sur le prix du vin, voir les remarques de Gras (1985, p. 373). C. Vandermersch $(1994$, p. 110) calcule, pour Hèrakleia au IV's., une rentabilité moyenne de 750 à 1250 drachmes à l'hectare, soit nettement plus que les céréales.

80. La tradition de la richesse que procurait à Marseille la vente de son vin est restée vivace jusqu'au $V^{\mathbf{c}} \mathrm{s}$. de notre ère si l'on en croit Paulin de Pella : « vineta, quibus solis urbis utitur ipsa omne ad praesidium vitae aliunde parandum" (les vignobles, qui sont les seules richesses dont use cette cité pour se procurer au dehors ce qui est nécessaire à la vie) (Eucharisticos, v. 526-527).

81. Cf. Lagrand, Thalmann, 1973, pl. XXX, ns 2-4 ; Bertucchi, 1992, p. $126-127$.

82. Une des amphores locales du Pègue était utilisée comme conteneur de céréales, ce qui semble bien avoir été sa fonction primaire (Bertucchi, 1992, p. 185-186). Sur la réutilisation des amphores de Marseille dans le même but, voir Chausserie-Laprée et al., 1988, p. 77-78.
Rappelons par ailleurs que les hypothèses de fabrication d'amphores micacées hors de Marseille, qui auraient pu témoigner d'une certaine décentralisation de la production massaliète, sont aujourd'hui abandonnées par la plupart des chercheurs ${ }^{83}$. Quant à l'idée de la diffusion de vin dans d'autres conteneurs, tels outres ou tonneaux que l'on ne repérerait pas parce qu'ils sont périssables, elle n'a reçu jusqu'à présent aucune confirmation ${ }^{84}$.

Dans la majorité des sites indigènes où une activité viticole est attestée par les pépins, la production paraît minime. Dans la plupart des échantillons en effet (à Mailhac, à Gailhan, au Marduel, à Pierredon, mais aussi aux Baux-de-Provence, au Baou-Roux, à Entremont, à Mouriès, à Mauressip, à Nages, à La Lagaste, etc.), le fait que quelques pépins de raisin soient mêlés à des quantités bien supérieures de grains de céréales a même porté à douter de l'existence même d'une production de vin.

Les concentrations de pépins évoquant des résidus de moût observées à Martigues et à Coudounèu ne concernent, dans les deux cas, qu'un vase isolé de petite capacité parmi des dizaines de conteneurs de céréales ou de légumineuses: quand bien même on aurait là les traces de fabrication de vin, ce qui semble tout à fait possible, il ne peut s'agir que de quantités limitées correspondant à une production ponctuelle, insuffisante en tout cas pour concurrencer les importations de vin marseillais, qui, au demeurant, et malgré la proximité de Marseille, apparaissent elles-mêmes comme assez faibles sur les deux sites en question. Il est difficile, dans ces exemples, de donner à la viticulture locale un véritable rôle dans l'économie des populations correspondantes. Rappelons par ailleurs que la viticulture n'est pas attestée très loin dans l'intérieur du pays: l'absence de pépins

83. L'idée d'une décentralisation de la production de Marseille est née des analyses conduites par J.-C. Échallier sur les inclusions contenues dans la pâte des amphores (voir notamment Échallier, 1982, 1983). Les conclusions de cet auteur ont cependant été contestées tant par les archéologues que par les analystes : on consultera à ce propos les deux dossiers consacrés à ce problème dans Documents d'Archéologie méridionale, 8, 1985 et Archéologie du Midi méditerranéen, 12, 1986.

84. Qu'il s'agisse du transvasement du vin importé pour la redistribution en pays indigène ( $c f$. par exemple Garcia, 1990, p. 116), ou de l'écoulement d'une production excédentaire ou spéculative. Sur cette question, la pseudo-« embouchure d'outre " en céramique grise monochrome présentée (avec un point d'interrogation) par Arcelin-Pradelle, 1984, fig. 52, $n^{\circ} 436$ (en fait probablement un support de vase) paraît un bien faible indice. 
dans les restes analysés au Pègue par exemple peut être en ce sens tenue pour significative (Lagrand, Thalmann, 1973, p. 30-31 ; Marinval, 1988a).

On ignore ce qu'il en est du Languedoc occidental, qui connaît à l'âge du Fer un développement considérable de l'agriculture céréalière, fondement d'un enrichissement certain des communautés protohistoriques littorales (Garcia, 1987 ; Py, 1993, p. 220-221) : ici, l'ancienneté des fouilles et l'absence de prélèvements et d'analyses empêchent pour l'heure d'évaluer correctement la place de la viticulture indigène dont on pressent l'existence, comme on l'a vu, à travers des témoignages indirects. Les recherches actuellement reprises, comme à Montlaurès ${ }^{85}$, pourraient amener prochainement des nouveautés dans ce domaine.

Reste le cas de Lattes, seul site où l'on dispose actuellement d'un faisceau d'informations suffisant pour dépasser les simples suppositions ${ }^{86}$. Les travaux pluridisciplinaires menés sur ce gisement ont en effet permis de disposer d'une périodisation des données carpologiques qui, comparée à d'autres types d'informations, apporte un éclairage nouveau sur la place de la viticulture dans l'économie indigène.

La courbe diachronique des pourcentages de pćpins de raisin parmi les restes de plantes cultivées montre en effet plusieurs paliers (fig. 4, A) :

- $A u I^{c}$ s. et au début du III's., les valeurs moyennes sont relativement faibles (entre 18 et $30 \%$ ), quoique bien supérieures à celles observées sur les autres sites de Gaule méridionale. Ces chiffres indiquent certainement l'existence d'une viticulture régulière, qui semble en place dès le début du V ${ }^{e}$ s., si l'on en croit les découvertes antérieures (Erroux, 1974), mais qui reste encore peu développée ${ }^{87}$.

85. Cf. Chazelles, 1997, 1999. Il semble néanmoins que les attestations de raisin soient, comme en Provence, très rares dans l'intérieur des terres : quelques unités seulement à Mailhac (Marinval, 1988a) ou à La lagaste (Erroux, 1980b) par exemple, et encore à date tardive ( $\mathrm{I}^{\mathrm{er}} \mathrm{s}$. avant notre ère).

86. Sur la viticulture lattoise, on consultera Py, 1990a, p. 83-84 ; Py, Garcia, 1993, p. 52-53 ; Buxó i Capdevila, 1989, 1992, 1997, p. 291-299. Synthèse des données (notamment carpologiques) dans Buxó i Capdevila, 1996.

87. Les chiffres relevés pour cette période (de 13 à $27 \%$ de pépins dans les échantillons de graines) ne sont cependant pas négligeables. À titre de comparaison, rappelons que pour le site lucanien de Roccagloriosa, C. Vandermersch $(1994$, p. 37) considère que les $30 \%$ de Vitis retrouvés parmi 800 restes végétaux du IV ${ }^{e}$ s. " attestent la pratique d'une arboriculture intensive ".
- Au milieu du III ${ }^{e}$ s. (entre 275 et 225 environ), on assiste à une première croissance des taux de pépins, qui s'établissent entre 40 et $45 \%$ des graines de plantes cultivées.

- À la fin du III ${ }^{\mathrm{e}}$ s. a lieu une nouvelle et forte croissance suivie d'une domination durable, les pourcentages de pépins recueillis dans l'habitat s'établissant entre 75 et $85 \%$ de 225 à 125 avant notre ère, et entre 92 et $99 \%$ à la fin du II ${ }^{\mathrm{e}}$ et au $\mathrm{I}^{\mathrm{er}} \mathrm{s}$. avant notre ère. De tels chiffres nous avaient conduits à supposer une expansion de la viticulture lattoise à partir de la fin du III $\mathrm{s}$. (loc. cit. supra). Or cette déduction vient de recevoir une confirmation éclatante par la découverte de traces de plantations extensives de vigne aux portes même de la cité, datables précisément de cette période.

Deux comparaisons permettent d'asseoir une interprétation de ces chiffres dépassant le simple constat.

D'une part, si l'on surimpose la courbe des pépins à celle des proportions d'amphores dans le mobilier (fig. $4, \mathrm{~B})^{88}$, on constate que la première est l'inverse de la seconde : la période de fréquence minimale des pépins correspond à la période de diffusion maximale des amphores, tandis que la croissance des proportions de pépins est synchrone avec un tassement progressif mais très net des taux d'amphores (massaliètes et italiques cumulées). Si l'on traduit la fréquence des pépins en terme de production vinicole et la fréquence des amphores en terme de volume d'achat de vin, on peut en conclure que le développement d'une viticulture extensive par les Lattarenses est lié à une relative raréfaction du vin sur le marché.

D'autre part, si l'on compare les proportions de pépins de raisin à celles de fragments de dolia retrouvés dans l'habitat (fig. 4, D), on constate que jusque vers 125 avant notre ère, les courbes sont très semblables (taux stables jusque vers 225, puis forte croissance), tandis qu'ensuite elles se séparent, les pépins restant très fréquents au $\mathrm{I}^{\mathrm{er}} \mathrm{s}$. avant notre ère alors que les dolia retrouvent les pourcentages des $\mathrm{IV}^{\mathrm{e}}-\mathrm{III}{ }^{\mathrm{c}} \mathrm{s}$. Interpréter le surplus de dolia constaté entre 225 et 125 comme un trait lié au développement de la viticulture ne pose pas de problème majeur, vu le rôle que l'on a reconnu à ces vaisseaux dans l'élaboration et dans la conservation du

88. Cette courbe, comme celle des dolia (infra) est obtenue par le comptage de la totalité des fragments de céramique recueillis en fouille, seule approche quantitative réellement objective. 
Fig. 4 - Évolution comparée des proportions de pépins de raisin parmi les graines de plantes cultivées et des proportions d'amphores et de dolia dans le mobilier archéologique sur le site de Lattes (Hérault).
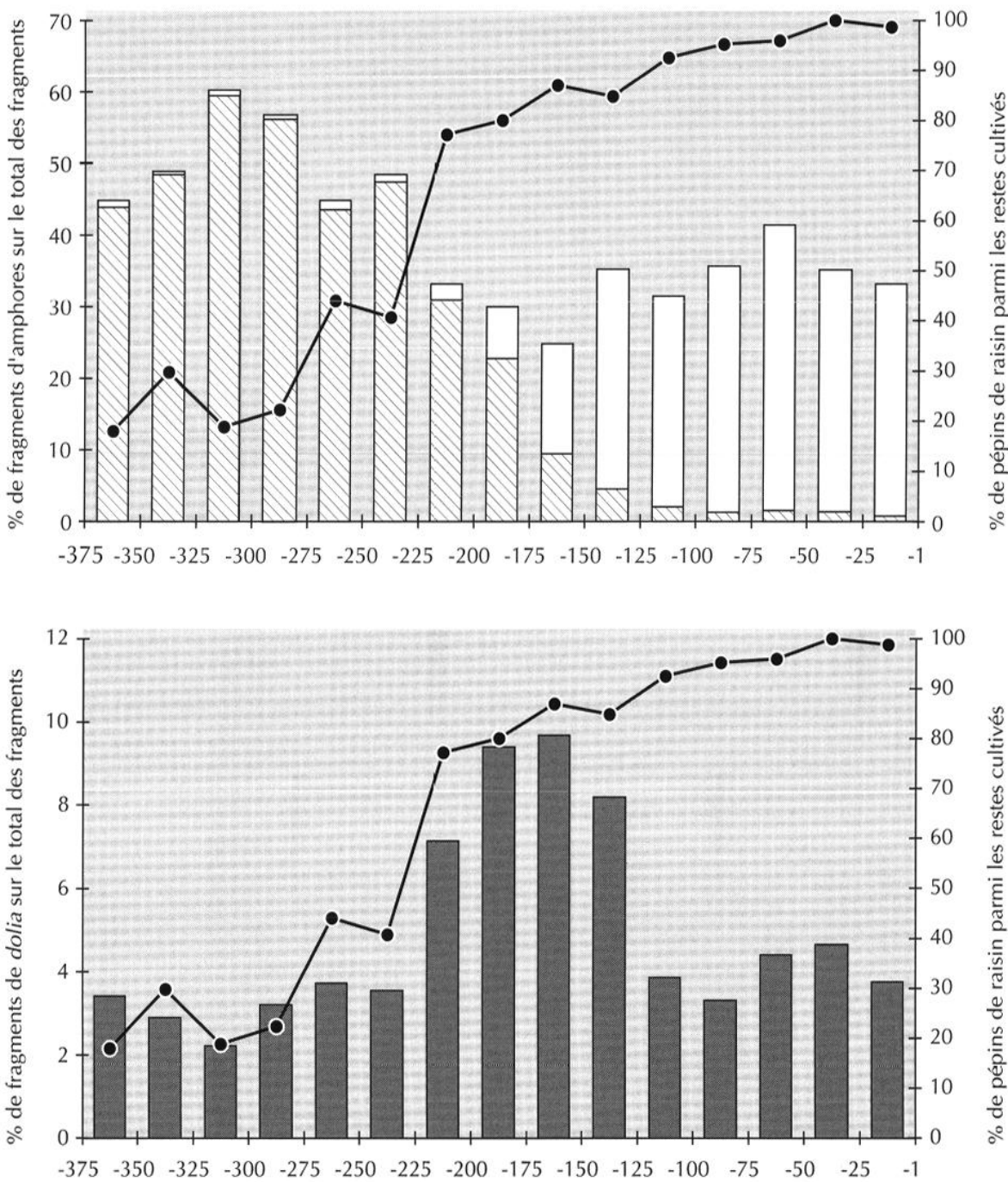

$\bullet$ - A : pépins $\square$ B : amphores $\quad$ C : massaliètes $\square$ D : dolia vin. Cependant, retenir cette hypothèse entraîne à supposer, pour la fin du $\mathrm{II}^{\mathrm{e}}$ et le $\mathrm{I}^{\mathrm{er}} \mathrm{s}$. avant notre ère où les courbes divergent, une délocalisation de l'élaboration et du stockage du vin, que l'on envisage la spécialisation de quartiers périphériques ${ }^{89}$ ou la création de fermes sur le territoire agricole ${ }^{90}$.

89. De la même façon qu'apparaissent à cette époque à la périphérie de la ville des quartiers où l'artisanat semble se concentrer (par exemple dans le secteur du sondage 26 du GAP);

90. Sur le dynamisme de l'occupation rurale entre la conquête et César dans la cité de Nîmes, voir le tableau brossé à partir des prospections de surface par Durand-Dastès et al., 1998, p. 88-98. L'importance du phénomène sur le territoire de Lattes est certainement masquée par le
Les données issues des fouilles de Lattes permettent de pousser plus loin le raisonnement et d'avancer, avec toute la prudence requise, sur le terrain social. En effet, le rôle des dolia dans la gestion des réserves de vin destinées à la consommation locale peut être valorisé aussi, sur ce site, par la découverte de salles spécialisées, que l'on a interprétées peut-être un peu vite comme des greniers ${ }^{91}$, mais qui pourraient bien correspondre plutôt

fort alluvionnement de la plaine littorale, comme le fait remarquer C. Raynaud dans le Bilan scientifique de la région Languedoc-Roussillon 1998, p. 174.

91. Cf. Py, López, 1990, p. 241-242 ; Garcia, 1992b, p. 180. 


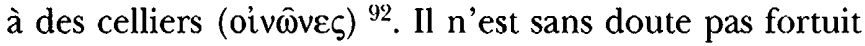
que de telles structures, qu'elles soient indépendantes et placées à l'extrémité d'un îlot (comme la salle 1 de l'îlot 4-nord vers 150-125) ou bien intégrées dans des demeures que leur taille ou leur plan mettent en marge des habitations ordinaires (maison 116 vers 225-200 et surtout maison 901 vers $175-125$ et maison 1605 vers 200-150) ${ }^{93}$, se situent toutes dans la phase de développement de la viticulture locale. La présence systématique de tels celliers dans les maisons à cour interprétées comme l'habitat d'une élite ${ }^{94}$ pourrait, si l'on retenait une telle interprétation, permettre de relier le développement de la viticulture à une accentuation de la différenciation sociale sur des bases économiques.

Il faut pour finir envisager la portée historique d'une telle documentation. La chronologie de l'apparition de la viticulture dans le triangle bas-rhodanien ne laisse guère de doute quant au rôle de la colonisation méditerranéenne dans cette innovation. Que les Grecs, et notamment les Marseillais, s'en soient attribués le mérite n'étonnera guère. Pourtant le cas de Lattes, où les traces de viticulture sont à la fois parmi les plus anciennes et les plus importantes en dehors de Marseille, invite à envisager la possibilité d'autres apports; on a en effet montré sur ce site la forte probabilité de la présence d'Étrusques au moment de la fondation de la cité (Py, 1995), et rien n'empêche de penser que ceux-ci ont pu également contribuer à la transmission d'une technique qu'ils maîtrisaient parfaitement ${ }^{95}$.

Le développement du commerce de Marseille entre la fin du $\mathrm{VI}^{\mathrm{e}}$ et le $\mathrm{IV}^{\mathrm{e}} \mathrm{s}$., illustré par la diffusion de ses amphores, a semble-t-il dans un premier temps limité l'extension de la viticulture locale. La logique des relations mises en place par Marseille à cette époque avec

92. Certaines de ces pièces de stockage présentent une organisation compatible avec les modèles de cellicrs connus dans le monde classique, notamment le fait que les jarres y soient un pcu cnterrées (se souvenir que Pline l'Ancien, $H . N$., XIV, 132, indique que les jarres à vin doivent être plus ou moins enterrées selon le climat local) et qu'clles soient disposées de manière très serrée, paroi contre paroi (voir notamment le cas de la salle 1 de l'îlot 4-nord), ce qui suppose une circulation par-dessus pour puiser.

93. Maison 116 (Py, 1996b, p. 162-163) ; maison 901 (ibid., p. 204-207); maison 1605 (ibid., p. 213-215).

94. (f. Py, 1996b, p. 252 ; Garcia, 1996.

95. Sur le vin étrusque, cf. Bouloumié, 1981, 1983b ; Torelli, 1981, p. $66 s q q$. les populations gauloises méridionales est fondée sur l'échange de produits complémentaires, vin contre céréales, avec toutes les conséquences que l'on sait sur l'évolution structurelle du monde indigène (Arcelin et al., 1982 ; Py, 1990b, 1993). Dans cette conjoncture, on peut envisager l'existence d'une viticulture indigène plus ou moins limitée, dont témoigneraient les restes observés à Lattes, Martigues et Coudounèu (concentrations de pépins, raflc, ceps...), mais aussi peut-être au Marduel, à Gailhan, à Pierredon... (pépins assez nombreux). Cette production marginale en effet n'était guère à même de concurrencer le vin grec, ni quantitativement ni sans doute qualitativement ${ }^{96}$.

La situation semble différente, du moins à lattes, aux III $^{\mathrm{e}}-\mathrm{I}^{\mathrm{er}} \mathrm{s}$., où se met en place de manière précoce une viticulture extensive (illustrée à la fois par les informations recueillies dans l'habitat et sur son territoire) dont on ne connaît pour l'heure l'équivalent qu'à la période romaine. La conjonction de ce processus et du retrait des amphores massaliètes (fig. 4, C) pose la question du sens d'une relation qui paraît évidente : il n'est pas facile de savoir en effet, dans l'état actuel des connaissances, si c'est la baisse de l'offre marseillaise (compensée seulement en partic par les apports italiens) qui ouvre des possibilités nouvelles à la viticulture locale ou nécessite son extension, ou bien si c'est cette extension qui provoque une baisse des achats extérieurs. L'isolement du cas de Lattes (si tant est qu'il ne s'agisse pas d'une situation provisoire) porterait plutôt à favoriser la première hypothèse, tandis que l'aspect graduel de la baisse des importations d'amphores entre la fin du $\mathrm{III}^{e}$ et la fin du $\mathrm{II}^{\mathrm{e}} \mathrm{s}$. pourrait conforter la seconde. Il faudrait se demander aussi quel fut le rôle des Marseillais eux-mêmes dans cette évolution, en tenant compte de leur présence possible, à cette époque, dans la cité de Lattara $^{97}$.

96. À propos de la qualité médiocre du vin de production localc par rapport aux vins grecs et italiens, citons Strabon parlant des Ligures : "Ils acheminent sur le marché de Gênes du bois, des peaux et du miel, qu'ils ćchangent contre de l'huile et du vin d'Italie, car le vin qu'ils produisent est pcu abondant, résineux et âpre au goût. " (Strabon, Géographie, IV, 6, 2).

97. La présence à demeure d'un petit contingent de courtiers grecs, et plus précisément massaliètes, dans l'habitat de Lattes aux IV"-l'rrs. avant notre ère a étć plusieurs fois évoquée à propos de l'étude de l'habitat, des céramiques (notamment de la vaisselle de cuisine grecque, couramment utilisée sur le site alors qu'elle fait complètement défaut dans les gisements indigènes de la région, $c$. Bats, 1992, p. 287) et des 
La même question se posera d'ailleurs encore dans cette région à l'époque de la romanisation (entre 125 et notre ère), où se mettent en place progressivement d'autres structures de production, liées à l'arrivée de colons italiens et à l'implantation de vastes cadastrations. La relative indépendance de Marseille jusqu'à sa chute en 49 a pu permettre à la cité de maintenir dans les limites de son imperium ${ }^{98}$ un rôle de contrôle et d'initia-

graffites (abécédaires, mots grecs, anthroponymes possiblement grecs, cf. Bats, 1988b).

98. Selon l'expression de Cicéron, De Officiis, II, 8, 28. tive $^{99}$. Le triangle bas-rhodanien aurait ainsi échappé aux limitations imposées par Rome au développement d'une viticulture indigène ${ }^{100}$, qui n'ont apparemment, si l'on se réfère aux données de Lattes, aucun impact sur la situation locale.

99. Voir sur ce point, en dernier lieu, Durand-Dastès et al., 1998, p. $90-93$.

100. Cicéron, De Republica, III, 9, 15-16. Sur la portée de ces mesures, discussion notamment dans Aymard, 1948, 1967 ; Clavel, 1970, p. 310-312 ; Van Rinsveld, 1981. 